\title{
A detailed dust energy balance study of the Sombrero galaxy
}

\section{Ilse De Looze, Maarten Baes, Jacopo Fritz, Gianfranco Gentile and Joris Verstappen}

Universiteit Gent, Sterrenkundig Observatorium

Krijgslaan 281, S9, 9000 Gent

email: ilse.delooze@ugent.be

\begin{abstract}
The Sombrero galaxy (M104) is an interesting object for a dust energy balance study due to its very symmetric dust lane, its proximity and its (nearly edge-on) inclination of $84^{\circ}$. From a panchromatic radiative transfer analysis, including scattering, absorption and thermal dust re-emission, we construct a standard model for M104 accounting for observations in the optical wave bands (stellar SED, images and extinction profiles in the $V$ and $R_{C}$ band). This standard model underestimates the observed dust emission at infrared wavelengths by a factor of $\sim 3$, similar to the discrepancy found in other energy balance studies of edge-on spirals. Supplementing this standard model with a young stellar component of low star formation activity in both the inner disk (SFR $\sim 0.21 \mathrm{M}_{\odot} \mathrm{yr}^{-1}$ ) and dust ring (SFR $\sim 0.05 \mathrm{M}_{\odot} \mathrm{yr}^{-1}$ ), we are capable of solving the discrepancy in the dust energy budget of the Sombrero galaxy at wavelengths shortwards of $100 \mu \mathrm{m}$. To account for the remaining discrepancy at longer wavelengths, we propose a secondary dust component distributed in quiescent clumps. This model with a clumpy dust structure predicts three-quarters of the total dust content to reside in compact dust clouds with no associated embedded sources.
\end{abstract}

Keywords. radiative transfer - ISM: dust, extinction - galaxies: M104

\section{Introduction}

Although dust grains make up only a small part of a galaxy's interstellar medium (ISM), they play an important role in several astrophysical processes. From optical studies, the extinguishing effect of dust grains on stellar light is evident. Alternatively, dust can be probed in emission at FIR/submm wavelengths. Ideally, a complementary study of the dust component from optical/UV images and its thermal re-emission at FIR/submm wavelengths allows to put strong constraints on the properties of dust in a galaxy. Edgeon spiral galaxies are particularly suited for detailed studies of their dust energy budget (Popescu et al. 2000, 2011, Misiriotis et al. 2001, Popescu \& Tuffs 2002, Alton et al. 2004, Dasyra et al. 2005, Baes et al. 2010). The edge-on view offers the opportunity to trace the dust out to large radii and to resolve the dust structure vertically, but also implies that all details of dust distribution vanish when integrating along the line-of-sight.

With an inclination of $\sim 84^{\circ}$, the Sombrero galaxy (M104), an early-type spiral (Sa), combines the advantages of edge-on galaxies with an inside view of the distribution of stars and dust in the inner disk. Along with its proximity $(D=9.2 \mathrm{Mpc})$, the large dataset of available FIR/submm observations (IRAS: Rice et al. 1988; MIPS: Bendo et al. 2006; LABOCA \& MAMBO: Vlahakis et al. 2008) and the very regular and symmetric shape of its dust lane, a spatially resolved analysis of the dust energy balance in M104 is possible. 


\section{Modeling approach and results}

To perform the radiative transfer modeling, we used the SKIRT code (Baes et al. 2003, 2011). SKIRT is a 3D Monte Carlo radiative transfer code that can model the dust extinction, including both absorption and scattering, and the thermal re-emission of dust, under the assumption of non local thermal equilibrium. Several advanced optimization techniques (stellar foam to generate random positions, forced scattering and continuous absorption) were implemented to improve the efficiency of the code.

To study the energy balance, we construct a model for the stars and dust in M104 based on optical data: SED fluxes from Dale et al. (2007), $V$ and $R_{C}$ band images from the Spitzer Infrared Nearby Galaxies Survey (SINGS) archive (Kennicutt et al. 2003) and $V$ and $R_{C}$ extinction curves from Emsellem (1995). Based on this optical model, the radiative transfer code SKIRT simulates the re-emission from dust at FIR/submm wavelengths. From a direct comparison of the predicted dust emissivity with infrared observations, one can study the energy budget in the Sombrero galaxy. In case of discrepancies, the optical model must be refined to balance the absorption of stellar light by dust grains and dust emission in this galaxy.

\subsection{Standard model}

For the stellar component in the bulge and disk, we adopt the axi-symmetric geometry from Emsellem (1995), who modeled the stars in M104 as a linear combination of 15 Multi-Gaussian Expansion (MGE) components. The luminosities of the MGE components were scaled linearly to fit the stellar SED. The shape of the stellar SED is parametrized by a Maraston et al. (2005) single stellar population (SSP) with an age of $10 \mathrm{Gyr}$ and a close to solar metallicity $Z=0.016$ (Vazdekis et al. 1997). For the dust component, a least-square fitting procedure to the observed $V$ and $R_{C}$ band images and extinction curves results in a dust ring modeled as a linear combination of 4 Gaussian rings:

$$
\rho_{d}(R, z)=\sum_{i=1}^{4} \rho_{0, i} \exp \left[-\frac{\left(R-R_{i}\right)^{2}}{2 \sigma_{i}^{2}}\right], \quad-\frac{h_{z}}{2} \leqslant z \leqslant \frac{h_{z}}{2} .
$$

where $\rho_{0, i}, R_{i}$ and $\sigma_{i}$ correspond to the relative mass contribution, radial distance to the maximum value and width of the Gaussian function, respectively, and $h_{z}=20 \mathrm{pc}$. The dust in the inner disk is distributed in an exponential disk $\left(h_{R}=2000 \mathrm{pc}, h_{z}=20 \mathrm{pc}\right)$, in correspondence to the model by Bendo et al. (2006). The total amount of dust is found to be $M_{d}=7.9 \times 10^{6} \mathrm{M}_{\odot}$, of which $M_{d}=7.5 \times 10^{6} \mathrm{M}_{\odot}$ is distributed in the dust ring and the remaining $M_{d}=4.1 \times 10^{5} \mathrm{M}_{\odot}$ resides in the inner disk. The nucleus, famous for its strange FIR/submm SED (Bendo et al. 2006, Vlahakis et al. 2008), is modeled as a point source, with an intensity field obtained through interpolation between the observed fluxes from the nucleus at IR/submm wavelengths.

When comparing the FIR/submm emission predicted from radiative transfer calculations for this standard model to the observed quantities, our model is found to underestimate the true FIR/submm emission by a factor of $\sim 3$ (see Figure 1, left panel). This "dust energy balance problem" is similar to what has been found in dust energy balance studies for several edge-on spirals (Popescu et al. 2000, Misiriotis et al. 2001, Popescu \& Tuffs 2002, Alton et al. 2004, Dasyra et al. 2005, Baes et al. 2010).

\subsection{Model with embedded star formation}

In an attempt to solve the discrepancy in the energy budget for the Sombrero galaxy, we adjust the standard model from the previous section by adding a young stellar population with a constant, continuous star formation during the past $100 \mathrm{Myr}$, distributed in the 
inner disk and the inner part of the dust ring. The shape of the SED is parametrized as a Starburst SED, generated from data of the Starburst99 library (Leitherer et al. $1999)$, with the same metallicity $(\mathrm{Z}=0.016)$ as the old stellar population (10 Gyr) in the Sombrero galaxy. A least-square fitting procedure to the observations at 24 and $70 \mu \mathrm{m}$ result in a young stellar component with a modest $\mathrm{SFR} \sim 0.05 \mathrm{M}_{\odot} \mathrm{yr}^{-1}$ in the dust ring and a more active component $\left(\mathrm{SFR} \sim 0.21 \mathrm{M}_{\odot} \mathrm{yr}^{-1}\right)$ in the inner disk. The total star formation activity $\left(\mathrm{SFR} \sim 0.26 \mathrm{M}_{\odot} \mathrm{yr}^{-1}\right.$ ) in our model agrees well with the SFR estimate based on $\mathrm{H} \alpha$ and radio observations (Kennicutt et al. 2009). The dissimilarity between model and observations in the FUV and NUV wavebands (see Figure 1, middle panel) is likely a consequence of the highly obscured nature of the young stellar population, which should be more embedded in our model. The dust mass in the ring was increased by a small amount $\left(M_{d}=7.8 \times 10^{6} \mathrm{M}_{\odot}\right)$ to maintain the resemblance between the modeled and observed $V$ band extinction curve. While this model solves the discrepancy in the dust energy balance at infrared wavelengths shortwards of $100 \mu \mathrm{m}$, the SED and MIPS $160 \mu \mathrm{m}$ image (see Figure 1, middle panel, and 2) clearly show that this model with embedded star formation can not account for the IR emission at longer wavelengths.

\section{Discussion}

Keeping in mind the agreement of the SFR activity in our model with 24 and $70 \mu \mathrm{m}$ observations and SFR calibrations, we argue that an additional component of heavily extinguished star formation will not solve the remaining discrepancy at longer wavelengths. It seems that either an additional dust component or dust grains with optical properties diverging from Galactic values (e.g. Alton et al. 2004 and Dasyra et al. 2005) need to be invoked to explain the inconsistency in the energy balance. While high emissivity dust grains can influence the shape of the SED at some wavelengths, they are incapable of solving the difference in the integrated dust energy balance. With a view on the inner regions of M104, we can rule out an additional dust component associated to a second dust disk, similar to the works Popescu et al. $(2000,2011)$ and Misiriotis et al. (2001). The assumption of a dust component distributed in dense clumps, which are not associated to localized embedded sources, seems more likely and is supported by high resolution optical imaging (HST, Christian et al. 2003), revealing a clumpy dust structure. When fitting a single-component modified blackbody to the residual SED fluxes, we require a dust mass $M_{d}=2.0 \times 10^{7} \mathrm{M}_{\odot}$ at a temperature $T_{d} \sim 16.2 \mathrm{~K}$ (see Figure 1, right panel). This result is in contrast to energy balance studies of the late-type galaxies NGC891 (Bianchi 2008) and UGC4754 (Baes et al. 2010), which require an additional clumpy dust component associated to localized embedded sources to explain the discrepancy in their energy balance. This might be a first indication for a difference in the dust distribution for spiral galaxies along the Hubble sequence. While early-type spirals tend to
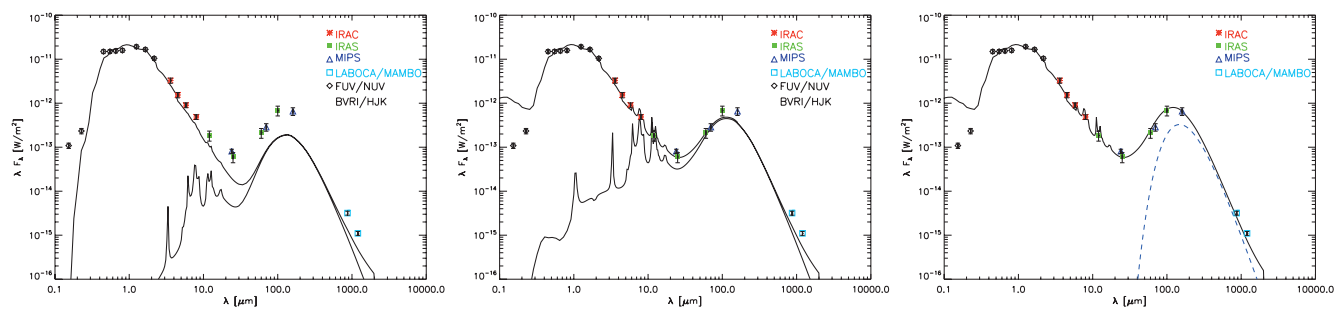

Figure 1. The total SED as obtained from SKIRT calculations (solid black line) for the standard model (left), model with embedded star formation (middle) and clumpy dust component (right). 


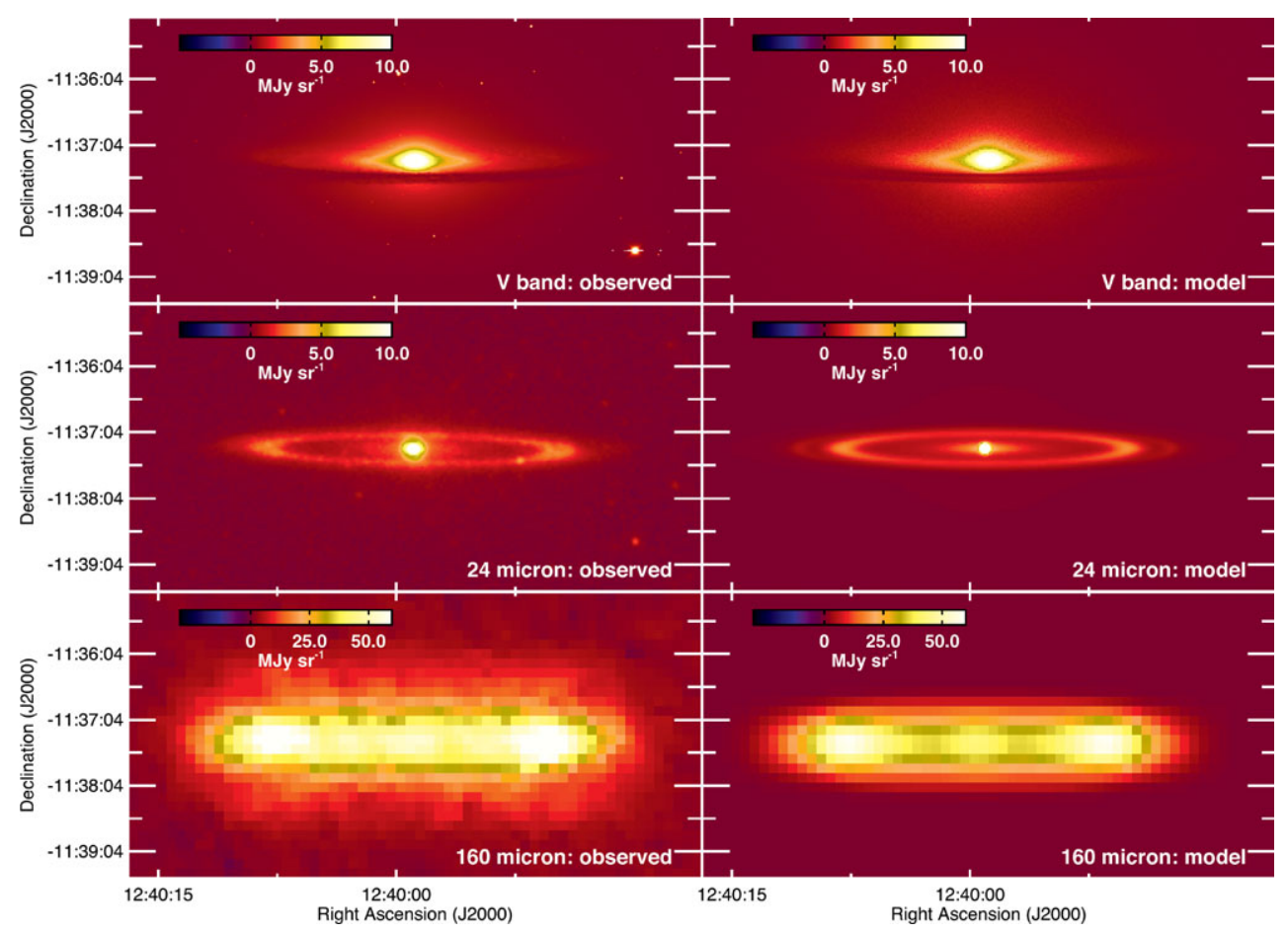

Figure 2. Results for the model with embedded star formation.

have the majority of dust distributed in quiescent clumps, most of the dust in late-type galaxies is associated to embedded localized sources.

\section{References}

Alton, P. B., Xilouris, E. M., Misiriotis, A., Dasyra, K. M., \& Dumke, M. 2004, A\& A, 425, 109 Baes, M., et al. 2010, MNRAS, 343, 1081

Baes, M., et al. 2010, A $\mho A$, 518, L39

Baes, M., Verstappen, J., De Looze, I. et al. 2011, ApJS, 196, 22

Bendo, G. et al. 2006, AJ, 645, 134

Bianchi, S. 2008, A\&\&A, 490, 461

Christian, C. A., Bond, H. E., \& Frattare, L. M. et al. 2003, Bulletin of the AAS, Vol. 35, p. 1398

Dale, D. A. et al. 2007, AJ, 655, 863

Dasyra K. M., Xilouris E. M., Misiriotis A. et al. 2005, in AIP Conf. Proc., Vol. 761, pp. 197-202

Emsellem, E. 1995, A\& A, 303, 673

Kennicutt, R. C., et al. 2003, PASP, 115, 928

Kennicutt, R. C., et al. 2009, ApJ, 703, 1672

Leitherer, C., et al. 1999, ApJS, 123, 3

Maraston, C. 2005, MNRAS, 362, 799

Misiriotis, A., Popescu, C. C., Tuffs, R., \& Kylafis, N. D. 2001, A\& $A$, 372, 775

Popescu, C. C., Misiriotis, A., Kylafis, N. D., Tuffs, R. J., \& Fischera J. 2000, A\&A, 362, 138

Popescu, C. C. \& Tuffs, R. J. 2002, MNRAS, 335, L41

Popescu, C. C., Tuffs, R. J., Dopita, M. A. et al. 2011, A\&A, 527, A109

Rice, W., Lonsdale, C. J., Soifer, B. T. et al. 1988, ApJS, 68, 91

Vazdekis, A., Peletier, R. F., Beckman, J. E., \& Casuso, E. 1997, AJ, 111, 203

Vlahakis, C., Baes, M., Bendo, G., \& Lundgren, A. 2008 A $\mathscr{E}$ A, 485, L25 


\section{Discussion}

WANG: I am wondering why you don't include a disk of old stars, which would solve the discrepancy at in near NIR without over-producing UV as you did when star formation was included instead.

DE Looze: A combination of a young and more evolved stellar population might be an option, but we don't think an old stellar population alone will solve the discrepancy at MIR wavelengths. Only young stars can provide enough UV emission to excite the PAHs. Furthermore, the amount of young stars we put in our model is consistent with several SFR tracers. Therefore, we argue that the young stars in our model should be more embedded, to solve the over-estimation of the UV emission in our model.

BENDO: I'd like to comment upon the use of a disk of evolved stars in radiative transfer models: In my follow-up work to Bendo et al. (2006), I found evidence for a near-infrared exponential disk that would suggest that a second evolved star component is needed in the model.

Clements: You noted an over prediction of UV \& suggested this could be solved by adding dust to star-formation regions. This would increase the far-IR. Would this solve the under-prediction of far-IR flux?.

DE Looze: The young stars will mainly heat the dust emitting at mid-IR wavelengths and will have a negligible contribution to the far-IR emission. In order to solve for the FIR/submm energy balance problem, the only solution seems to be an extra cold dust component distributed in quiescent clumps.

TuFfs: Following up on the comment from Dave Clements, I would also like to comment that a geometrically correlated clumpy distribution of stars and dust might also help to resolve the discrepancy between the model-predictions and observations in the UV, due to the stronger local coupling between the UV \& dust. Also, a potential difficulty of invoking "Passive" clumps, without stars, is that they would lead to high dust-to- gas ratios. What dust-to-gas ratio is implied by your model?

De Looze: When scaling the HI mass $\left(3.2 \times 10^{8} \mathrm{M}_{\odot}\right.$; Bejeje et al. 1984) and $\mathrm{H}_{2}$ mass (4.4 x $10^{8} \mathrm{M}_{\odot}$; Bejeje et al. 1991) with a factor of 1.4 to include Helium, we get a total gas content of $1.06 \times 10^{9} \mathrm{M}_{\odot}$ at a distance of $\mathrm{D}=9.2 \mathrm{Mpc}$. Our radiative transfer model, including the additional dust distribution in quiescent dust clumps, accounts for a total dust mass of $\mathrm{M}_{d}=2.8 \times 10^{7} \mathrm{M}_{\odot}$, implying a gas-to-dust ratio of $\sim 38$. Considering that the molecular gas has only been observed towards some directions in the ring and the total molecular gas mass was derived under the assumption of a constant ratio of the $\mathrm{H}_{2} / \mathrm{HI}$ surface mass density of 1.3 throughout the $\mathrm{HI}$ emission regions, we need to take into account a large uncertainty factor for this estimate of the gas-to-dust ratio in M104. 